\title{
INFLUENCIA DE LA ESTRUCTURA ARBÓREA EN LA PRODUCCIÓN DE PASTO EN UN SISTEMA DE ÁRBOLES DISPERSOS EN POTRERO, TIPITAPA, NICARAGUA
}

\author{
Alvaro Noguera-Talavera', Francisco Reyes Flores', Ivannia Murillo², Lea Sanchez ${ }^{2}$ \\ ${ }^{1}$ Docentes Investigadores Facultad de Recursos Naturales y del Ambiente \\ ${ }^{2}$ Egresadas Ingeniería Forestal.
}

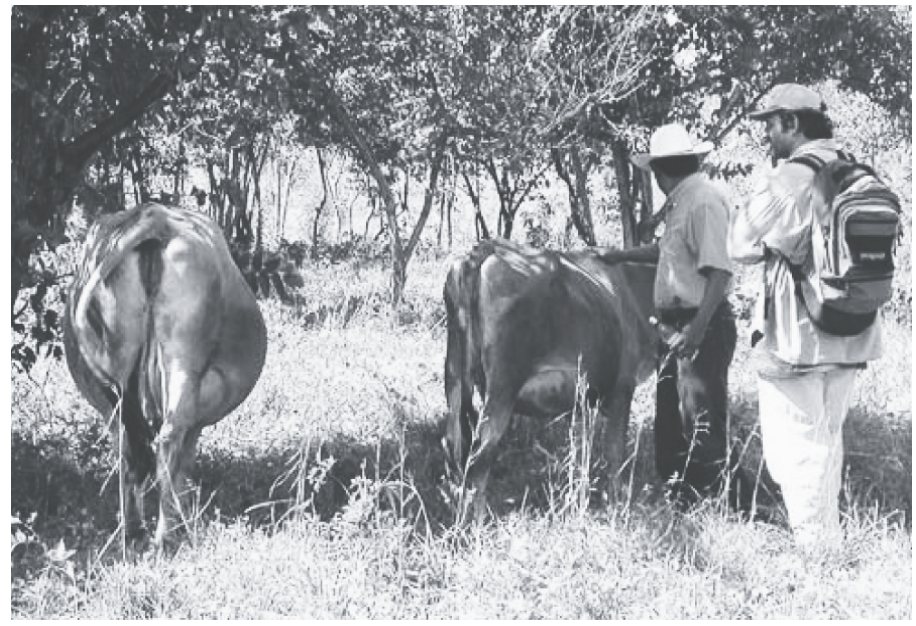

\section{RESUMEN}

En el Municipio Tipitapa, Departamento de Managua, en un sistema silvopastoril, se llevó acabo un estudio con el propósito de evaluar la influencia de la estructura de las arbóreas sobre algunas variables de la producción del pasto. Las arbóreas fueron caracterizadas a partir del cálculo de la densidad de individuos pertenecientes a las categorías fustales y brinzales, en parcelas de $20 \mathrm{~m}$ x $20 \mathrm{~m}$. Así mismo fueron tomadas variables de altura, diámetro y longitud de copa. Para el componente herbáceo (pasto) se evaluó la incidencia de las arbóreas a partir de la densidad del pasto en parcelas bajo sombra y parcelas a pleno sol; además de la variable, número y longitud de brotes. Se encontraron un total de 20 especies leñosas, de las cuales cuatro son útiles como forrajeras, siendo las restantes proveedoras de sombra y otras (3 especies) fijadoras de nitrógeno. Las especies más representativas con base en la abundancia relativa son: Tabebuia rosea (53.93\%), Pithecellobium dulce (26.35\%), Azadirachta indica (10.19\%). Las leñosas, en la categoría de fustales presentan densidades dentro del rango del número de árboles reportados en sistemas de árboles dispersos en potreros en Centroamérica .Los valores de diámetro y altura promedio son de $1.0 \mathrm{~cm}$. y $0.92 \mathrm{~m}$, respectivamente. El porcentaje de cobertura generado por las leñosas es de 10.6. Mediante análisis estadístico se determinó que no existen diferencias entre la densidad del pasto $(\mathrm{p}>0.05)$ entre parcelas bajo sombra. De la misma forma, no se encontraron diferencias $(\mathrm{p}>0.05)$

\section{SUMMARY}

A study of the effect of trees shade on grazing grounds productivity in a livestock system was carried out. The study was placed at Los Tercios, Tipitapa municipality, Managua. Tree density, distance between trees, tree diameter, tree crown length and plant (seedling and trees) high were evaluated in $20 \mathrm{~m}$ x $20 \mathrm{~m}$ plots. Herbaceous component (grazing) was evaluated trough plant density, buds number and buds long at under shadow and to full sun plots. Floristic composition includes 20 trees species. Four trees species are useful to fodder, others are useful to supply shade to animal component and to fix nitrogen in soil. The most representative species based on relative abundance are Tabebuia rosea (54\%), Pithecellobium dulce (26.4\%), and Azadirachta indica (10.2 $\%$ ). The mean density of high forest was calculated in 71 tress ha-1. This category have mean high and mean diameter of $6.9 \mathrm{~m}$ and $18.4 \mathrm{~cm}$ respectably. In the case of seedling category, the mean density is 1092 individuals ha ${ }^{-1}$, with a mean basal diameter of 1.0 centimeter and a mean high of 0.92 meter. Shade covering perceptual value supplied by trees was calculated in 10.6. Statistical analyze did no show difference $(p>0.05)$ between grass densities in plots under shadows. Statistical differences $(p<0.05)$ were found in grass density in plots under shadow located $10 \mathrm{~cm}$ of from the center of trees and grass density in plots open to sun. The analysis revealed statistical $(\mathrm{p}<0.05)$ differences in the case 
entre parcelas a $5 \mathrm{~m}$ y la densidad de parcelas a pleno sol. Se encontraron diferencias $(\mathrm{p}<0.05)$ entre la densidad de pasto en parcelas bajo sombra ubicadas a $10 \mathrm{~m}$ del centro de árboles y la densidad de pasto en parcelas a pleno sol. De la misma forma, el análisis reveló diferencias estadísticas $(\mathrm{p}<0.05)$ en cuanto al número y longitud de brotes y correlación entre las variables área de copa y abundancia de las arbóreas con las variables número de brotes y longitud de brotes. of buds number and buds longitude and correlation between the variable tree crown area and tree abundance with the variable number of buds and longitude of buds.

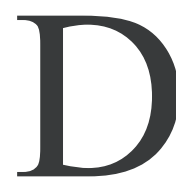
e acuerdo con Esquivel et al., (2003), el establecimiento de pasturas en Centroamérica ha seguido la dinámica de la agricultura migratoria. Es por ello que los beneficios de la conversión del bosque tropical a pasturas son temporales ( \pm 5 años). Lo anterior se debe principalmente a la perdida de nutrientes, compactación y erosión del suelo que resultan en la degradación de la pastura.

Los sistemas silvopastoriles son una modalidad de agroforestería pecuaria que combina los pastos para ganadería con árboles y arbustos. Estos sistemas cumplen algunas funciones de los bosques naturales porque poseen vegetación permanente con raíces profundas y un dosel denso. Son una alternativa real al tipo de ganadería que prevalece en América Latina, generan servicios ambientales y mejoran la calidad de vida de los productores y de las familias que dependen de las fincas ganaderas para su sustento (CATIE, 2004).

En la dinámica de los sistemas silvopastoriles es determinante la interacción de plantas leñosas perennes, la vegetación herbácea, los bovinos u ovinos y el suelo. Es por ello que algunos estudios han demostrado que la gramínea debajo de la copa de los árboles es sometida a cambios sustanciales en la cantidad y calidad de la luz que recibe (Pezo \& Ibrahim 1998; Plevich et al., 2002). Sin embargo, aun cuando la cantidad de biomasa producida por la gramínea se reduce con el sombreo (Plevich et al., 2002; Betancourt et al., 2003), el área foliar y la eficiencia fotosintética incrementa en estas condiciones, lo que implica una mayor calidad del forraje producido en condiciones de sombra intermedia. De igual manera, la sombra del árbol reduce la temperatura foliar en la gramínea, lo que ocasiona una menor transpiración, aumentando la eficiencia de uso de agua de la gramínea (Plevich et al., 2002; Gil et al., 2005).

A pesar del conocimiento adquirido hasta ahora, en Nicaragua se ha estudiado poco la relación entre componentes de sistemas silvopastoriles; por lo que se ha identificado la necesidad de entender el funcionamiento de estos sistemas para desarrollar estrategias flexibles de manejo tendiente a su utilización sostenible. Este trabajo plantea como objetivo conocer la influencia de las arbóreas sobre la producción del componente pasto.

\section{MATERIALES Y MÉTODOS}

El estudio se realizó en la finca Los Tercios en el Departamento de Managua, en el Municipio de Tipitapa, Comunidad Los Tercios. El área presenta una altitud promedio de 60 metros sobre el nivel del mar, un clima que va de sabana herbácea tropical a bosque seco caducifolio, con una precipitación que oscila entre los $1000 \mathrm{~mm}$ y $1250 \mathrm{~mm}$; y una temperatura promedio de $27.5^{\circ} \mathrm{C}$ (Servicio Forestal Nacional 1993).

Descripción de la recolección de datos. El estudio se desarrolló desde Enero a Noviembre del 2006. Los datos fueron recopilados en la época seca, entre los meses de Enero a Abril.

Con el fin de describir la composición botánica y la estructura de las leñosas arbóreas en el sistema; en cada potrero de la finca $(n=7)$ se delimitaron diez parcelas temporales de muestreo, seleccionadas de manera aleatoria. Las dimensiones definidas para cada parcela fue de $20 \mathrm{~m}$ x $20 \mathrm{~m}$. Se medió el diámetro a la altura del pecho (DAP $=1.3 \mathrm{~m}$ del suelo), la altura total y la longitud de copa de los árboles de la categoría fustal (DAP $\geq 10 \mathrm{~cm}$ ). Así mismo, se registró el diámetro basal, altura total y longitud de copa de los brinzales $(0.3 \mathrm{~m} \geq$ altura $<1.5 \mathrm{~m})$.

Se realizó un muestreo de la densidad, longitud y número de brotes de pasto estrella (Cynodon nlemfluensis) bajo la copa de diez árboles seleccionados de forma aleatoria. Mediante una modificación del "diseño anidado" descrito en Hernández et al., (2002) y Murillo y Sánchez (2007); bajo cada árbol se establecieron cuatro parcelas temporales; el área de cada parcela fue $1 \mathrm{~m}^{2}$. De las cuatro parcelas establecidas bajo la copa de los árboles, dos parcelas se establecieron a una distancia de 5 metros, y dos parcelas a una distancia de 10 metros; ubicadas considerando los puntos cardinales. La densidad presentada en la sección de resultados corresponde a valores medios por árbol. La longitud de cada brote (expresado en centímetros), se midió desde la base hasta el ápice utilizando una cinta métrica. Para calcular la 
densidad de brotes se tomó la mata y se contabilizó cada uno de los hijos (brotes).

Análisis de los datos. El cálculo del número de árboles por hectárea se hizo con base en la siguiente fórmula:

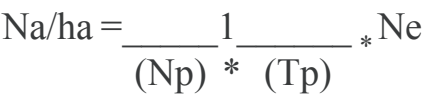

Donde:

Na/ha: Es el número de árboles por hectárea

Np: Es el número de parcelas muestreadas

Tp: Es el tamaño de la parcela expresado en hectárea

Ne: Es el número de árboles encontrados durante el inventario

Para los cálculos de cobertura de superficie de copa se empleó la fórmula siguiente:

$\mathrm{Ac}=\pi / 4(\mathrm{~s} \mathrm{~m})^{2}$

Donde:

Ac: Área de copa de un árbol

$\pi / 4: 0.7854$

S: Promedio de la proyección de copa en metro

Para conocer el porcentaje de área de cobertura del área Total se utilizó la siguiente fórmula:

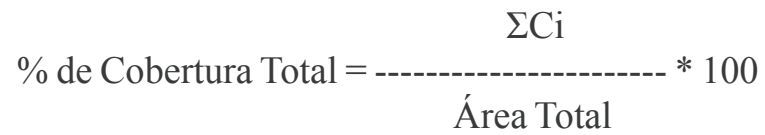

Con el objetivo de inferir en el efecto de las especies arbóreas sobre el pasto, se realizó una prueba estadística (análisis de $\mathrm{t}$ - student) con el programa INSTAT versión 3.0, partiendo de los siguientes supuestos: bajo la copa de los árboles a mayor distancia del fuste, mayor densidad de pasto y es mayor la densidad y longitud de brotes de pasto en sitios a pleno sol que en aquellos bajo la copa de los árboles.

Para evaluar el nivel de relación entre variables que modifican el efecto de la sombra en sistemas silvopastoriles (Pezo \& Ibrahim, 1998), se realizó un análisis de correlación simple con el programa estadístico INSTAT versión 3.0. Las variables confrontadas fueron: Área de copa $\left(\mathrm{m}^{2}\right)$, altura de los fustales y abundancia de las arbóreas versus las variables densidad del pasto (individuos $/ \mathrm{m}^{2}$ ), longitud de brotes y número de brotes del componente pasto.

\section{RESULTADOS Y DISCUSIÓN}

Inventario de las leñosas encontradas en el sistema de árboles dispersos en potreros. En el área de estudio (13.8 hectáreas) se cuantificó la presencia de 16060 individuos representantes de 20 especies arbóreas. De este total, 15080 individuos (1092 plantas $/ \mathrm{ha}^{-1}$ ) corresponden a la categoría brinzal, mientras 980 (71 árboles $/ \mathrm{ha}^{-1}$ ) pertenecen a la categoría fustal (Cuadro 1). La riqueza de especies por área de pastoreo presenta valores en un rango de 9 a 14. El número de especies por potrero es similar, presentando un valor medio de 10 especies. La diversidad de arbóreas por área de pastoreo presentó diferencias estadísticas $(\mathrm{p}<0.05)$. El índice de Shannon-Wienner reveló poca equidad en la abundancia de individuos por especie por potrero. La abundancia de arbóreas de ambas categorías en este trabajo coincide con estudios que relacionan la abundancia de las arbóreas con el nivel de degradación de las pasturas. En este sentido, Hernández et al., (2002), han reportado altas densidades de arbóreas en pasturas, con niveles de degradación clasificados como moderada a medianamente severos.

Las especies más representativas dentro del sistema, con base en la abundancia relativa son: Tabebuia rosea con $53.9 \%$, seguido de Pithecellobium dulce con 26.4 $\%$, y Azadirachta indica con $10.2 \%$. Entre los usos más importantes de las especies identificadas se pueden mencionar, sombra (100\% de las especies), forraje (25\%), cercas vivas (30\%), y leña (45\%).

El diámetro promedio para los fustales es de 18.4 centímetros, y se calculó una altura promedio de 6.9 metros. Para los individuos de la categoría brizal se calculó el diámetro promedio en un centímetro, y una altura promedio de 0.92 metros (Tabla 1 ).

El porcentaje de cobertura generado por las leñosas $(10.6 \%)$, sugiere un valor relativamente bajo; ya que a pesar de la existencia de una alta densidad de individuos en el área, el parámetro porcentaje de cobertura es bajo en relación al área total. Esto se debe a que la mayoría de los individuos por pertenecer a la categoría brinzal, poseen copas relativamente pequeñas que generan valores bajos de densidad de copa y por tanto baja cobertura al suelo. 
Tabla 1. Variables estructurales de las especies arbóreas presente en la Finca Los Tercios Tipitapa, 2007

\begin{tabular}{lll}
\hline Variables estructurales & $\begin{array}{l}\text { Valores de } \\
\text { las variables } \\
\text { estructurales }\end{array}$ & $\begin{array}{l}\text { Número (n) de } \\
\text { individuos }\end{array}$
\end{tabular}

\begin{tabular}{lrr}
\hline Diámetro promedio de brinzales $(\mathrm{cm})$ & 1.0 & 3058 \\
Diámetro promedio de fustales $(\mathrm{cm})$ & 18.4 & 200 \\
Altura promedio de brinzales $(\mathrm{m})$ & 0.9 & 3058 \\
Altura promedio de fustales $(\mathrm{m})$ & 6.9 & 200 \\
Porcentaje de cobertura (\%) & 10.6 & - \\
Densidad de brinzales (individuos $\left./ \mathrm{ha}^{-1}\right)$ & 1092.0 & - \\
Densidad de fustales (individuos $\left./ \mathrm{ha}^{-1}\right)$ & 71.0 & -
\end{tabular}

$\mathrm{n}$ : Es el número o valores de individuos encontrados en un área de muestreo de 2.8 hectáreas.

Influencia de las arbóreas sobre la producción del pasto. Diferencias estadísticas no significativas ( $\mathrm{p}>$ 0.05 ) fueron determinadas al comparar la densidad de pasto entre parcelas a diferente distancia ( $5 \mathrm{~m}$ y $10 \mathrm{~m}$ ) bajo la copa de los árboles. La densidad de pasto en parcelas bajo sombra fue significativamente diferente $(\mathrm{p}<0.05)$ a la densidad de pastos en las parcelas a pleno sol; presentando las últimas un mayor número de individuos por metro cuadrado (Tabla 2). menor longitud de brotes que la del pasto que se encontró en las parcelas a pleno sol (Figura 1).

De la misma forma, se obtuvieron diferencias estadísticas $(\mathrm{p}<0.05)$, aal comparar el número de brotes del pasto entre parcelas bajo la copa de los árboles y el pasto de las parcelas a pleno sol (Figura 2).

La sombra esta ejerciendo efecto sobre la longitud y número de los brotes de pasto. Este resultado coincide con lo expuesto por Villafuerte et al., (1999), en cuanto a

Tabla 2: Densidad promedio de individuos en parcelas bajo sombra y parcelas a pleno sol en un sistema de árboles dispersos en potreros en la finca Los Tercios, Tipitapa, 2007

\begin{tabular}{lll}
\hline $\begin{array}{l}\text { Número } \\
\text { de Parcela }\end{array}$ & $\begin{array}{l}\text { Densidad de pasto bajo sombra } \\
\text { (individuos por parcela) }\end{array}$ & $\begin{array}{l}\text { Densidad de pasto a pleno sol } \\
\text { (Individuos/Parcela) }\end{array}$ \\
& $5 \mathrm{~m}$ & $10 \mathrm{~m}$
\end{tabular}

\begin{tabular}{lrrr}
\hline 1 & 4 & 2 & 15 \\
2 & 1 & 0 & 15 \\
3 & 0 & 5 & 9 \\
4 & 8 & 11 & 18 \\
5 & 10 & 15 & 22 \\
6 & 27 & 18 & 46 \\
7 & 11 & 13 & 21 \\
8 & 0 & 0 & 12 \\
9 & 3 & 5 & 11 \\
10 & 8 & 9 & 23
\end{tabular}

Se encontraron diferencias estadísticas significativas $(\mathrm{p}<0.05)$, al comparar la longitud de los brotes de pasto en parcelas bajo sombra y la misma variable en parcelas a pleno sol. La sombra proporcionada por los árboles influye en la variable longitud de brotes del pasto, ya que el pasto encontrado bajo la copa de los árboles tiene que bajo sombra, muchos pastos presentan rendimientos que van de regulares a bajos, además de reducir la producción promedio de materia seca.

A pesar de ello, uno de los efectos benéficos de la sombra es que aumenta la calidad de las pasturas en cuanto a contenido de proteína (Pezo \& Ibrahim, 1998). 


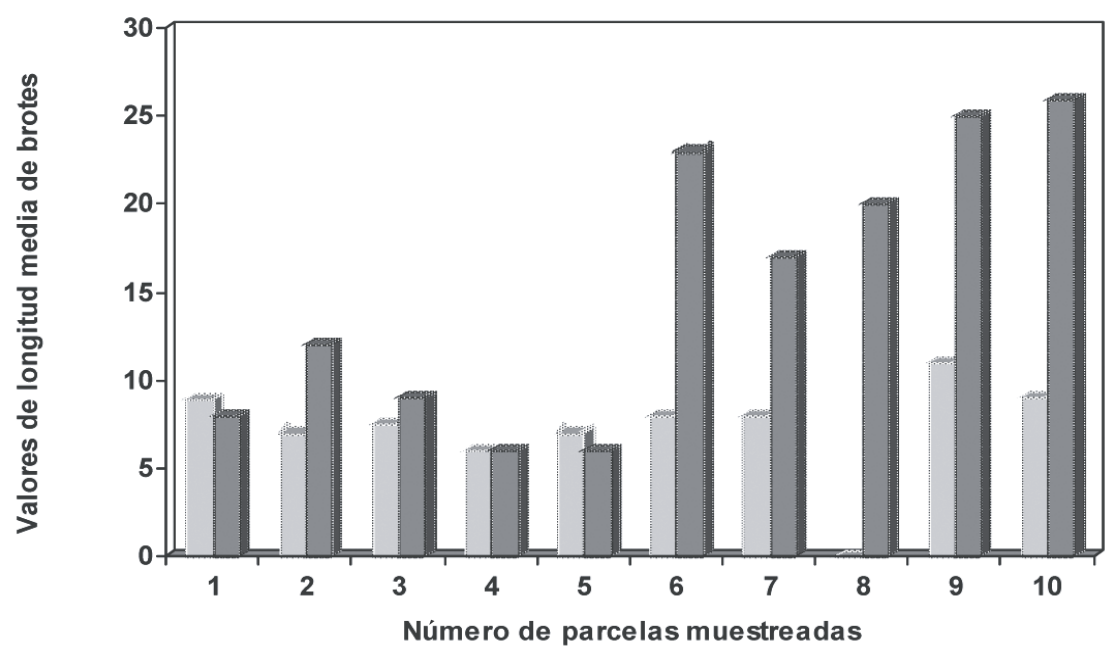

\section{LBPbSom $\square$ LBPaSol}

Figura 1: Longitud media de brotes (cm) en parcelas bajo sombra (LBPbSom) y parcelas a pleno sol (LBPaSol), en un sistema de árboles dispersos en potreros, finca Los Tercios, Tipitapa, 2007.

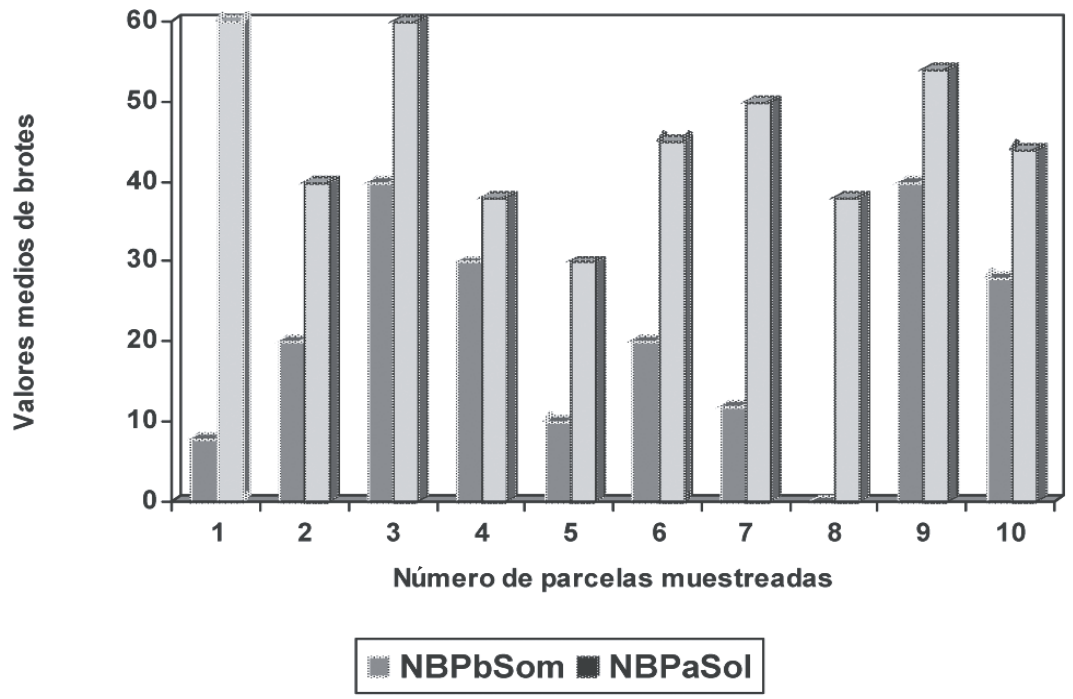

Figura 2: Número medio de brotes en parcelas bajo sombra (NBPbSom), y parcelas a pleno sol (NBPaSol), en un sistema de árboles disperso en potreros en la finca Los Tercios, Tipitapa, 2007.

Al analizar la correlación entre la variable altura del componente arbóreo, y las variables densidad de pasto, número promedio de brotes y longitud promedio de brotes, determinantes de la producción de pasto estrella (Cynodon nlemfluensis) se obtuvo una correlación de tipo inversa no significativa $(\mathrm{r}=-0.4535,-0.1466 \mathrm{y}$ -0.1373 , respectivamente).
En cuanto al área de copa y su relación con las variables densidad de pasto, número de brotes por mata y longitud de brotes; se determinó la no existencia de correlación para las primeras dos $(\mathrm{r} \approx 0)$ y un tipo de correlación negativa $(r=-0.1663)$ para la variable número de brotes. El comportamiento relacionado a las variables densidad de pasto y número de brotes observado en este trabajo difiere de los resultados presentados por Maya et al., 
(2005), quienes afirman que el pasto estrella tiene la habilidad de cambiar su hábito de crecimiento a medida que aumenta la cobertura arbórea; experimentando un incremento en la proporción de tallos erectos en lugar de tallos rastreros o estolones. Por el contrario existe la teoría generalizada que la sombra de las leñosas disminuye la producción del pasto; lo cual se comprueba al interpretar el valor del coeficiente de correlación; el que indica que un aumento del área de copa causa una disminución del número de brotes por mata. Tanto para la variable altura, como área de copa, la prueba de significancia ( $95 \%$ de confianza) reveló correlación no significativa entre las variables confrontadas.

Las variables abundancia de las arbóreas y densidad de pasto, presentan un tipo de relación positiva media $(\mathrm{r}=0.5141)$, mientras la abundancia de fustales versus número de brotes presenta correlación negativa media $(\mathrm{r}=-0.5361)$. Para abundancia de fustales y longitud brotes, el resultado revela una alta correlación de tipo positiva $(r=0.7581)$.

\section{CONCLUSIÓN}

Los parámetros de las especies leñosas de mayor influencia en la producción de pasto en el área de estudio son: el área de copa y la abundancia. El manejo del área debe ser orientado hacia una disminución en la abundancia de las arbóreas de la categoría brinzal, para disminuir así la competencia entre el pasto y la regeneración de las leñosas del sistema.

\section{REFERENCIAS BIBLIOGRÁFICAS}

Betancourt, K; Ibrahim, M; Harvey, CA; Vargas, B. 2003. Efecto de la cobertura arbórea sobre el comportamiento animal en fincas ganaderas de doble propósito en Matiguás, Matagalpa, Nicaragua. Agroforesteria en las Américas. 39-40: 47-51.

CATIE (Centro Agronómico Tropical de Investigación y Enseñanza, CR). 2004. Proyecto: Enfoques silvopastoriles integrados para el manejo de ecosistemas.

Esquivel, H; Ibrahim, M; Harvey, CA; Villanueva, C; Benjamin, T; Sinclair, FL. 2003. Árboles dispersos en potreros de fincas ganaderas en un ecosistema seco de Costa Rica. Agroforesteria en las Américas. 39-40:24-29.

Gil, JL; Espinoza, Y; Obispo, N. 2005. Relaciones suelo-planta-animal en sistemas silvopastoriles. Revista digital del Centro Nacional de Investigaciones Agropecuarias. 9:20-26. URL: www.ceniap.gov.ve/ceniaphoy/ articulos. Visitado el 06/03/2006.

Hernández, KJ; Ibrahim, M; Detlefsen, G; Harvey, C; Prins, K. 2002. Cuantificación y calificación de pasturas degradadas incorporando conocimiento local de ganaderos de la Calzada Mopan, Dolores, Petén, Guatemala. Agroforesteria en las Américas. 35-36: 62-68.

Maya, GE; Duran, CV; Ararat, JE. 2005. Altura, disponibilidad de forraje y relación hoja-tallo del pasto estrella solo y asociado con leucaena. Revista Agronómica. 54:2.

Murillo, GI; Sánchez, ML. 2007. Caracterización de un sistema silvopastoril: Efecto de las leñosas sobre el pasto en la finca "Los Tercios", Municipio Tipitapa.

Plevich, J; Nuñez, C; Cantero, J; Deaestri, M; Viale, S. 2002. Biomasa del pastizal bajo diferentes densidades de pino (Pinus elliottii). Agroforesteria en las Americas. 33-34: 19-23.

Pezo, D; Ibrahim, M. 1998. Sistemas silvopastoriles. Proyecto agroforestal. CATIE. Turrialba, Costa Rica. $258 \mathrm{p}$.

Servicio Forestal Nacional. 1993. Uso actual de la tierra con énfasis en la cobertura de bosque en 9 departamentos de Nicaragua. Managua, Nicaragua. pp.25.

Villafuerte, L, Arze, J; Ibrahim, M. 1999. Rendimiento de pasturas con y sin sombra en el trópico húmedo de Costa Rica. Agroforesteria en las Américas. 6:2-23. 54-56. 